\title{
Low hepatitis $B$ immunogenicity of a hexavalent vaccine widely used in Germany: results of the German Health Survey for Children and Adolescents, 2003-2006
}

\author{
P. JORGENSEN ${ }^{1,2}$, C. POETHKO-MÜLLER ${ }^{3}$, W. HELLENBRAND ${ }^{1}$, W. JILG ${ }^{4}$, \\ W. THIERFELDER ${ }^{3}$, C. MEYER ${ }^{1}$, M. AN DER HEIDEN ${ }^{1}$, M. SCHLAUD $^{3}$ \\ AND D. RADUN ${ }^{1 *}$ \\ ${ }^{1}$ Department for Infectious Disease Epidemiology, Robert Koch Institute, Berlin, Germany \\ ${ }^{2}$ European Programme for Intervention Epidemiology Training, European Centre for Disease Prevention and \\ Control, Stockholm, Sweden \\ ${ }^{3}$ Department for Epidemiology and Health Reporting, Robert Koch Institute, Berlin, Germany \\ ${ }^{4}$ Institute for Medical Microbiology and Hygiene, University Regensburg, Germany
}

(Accepted 12 February 2010; first published online 17 March 2010)

\section{SUMMARY}

The success of childhood vaccination against hepatitis B relies on persistence of immunity into adolescence and adulthood. In 2000, two hexavalent vaccines with a hepatitis B component $\left(\right.$ Hexavac ${ }^{\mathbb{R}}$, Infanrix hexa ${ }^{\mathbb{R}}$ ) were introduced in Germany. Hexavac was withdrawn in 2005 amidst concerns about its long-term hepatitis B protection. We compared hepatitis B surface antibody (anti-HBs) levels in children fully vaccinated with Hexavac or Infanrix hexa $(n=477)$ in a secondary data analysis of a large cross-sectional health survey in Germany. On average $2 \cdot 4$ years after vaccination, $25 \cdot 3 \%$ of Hexavac vaccinees had anti-HBs levels $<10 \mathrm{mIU} / \mathrm{ml}(95 \% \mathrm{CI}$ 19.0-32.8) compared to $4 \cdot 7 \%$ of Infanrix hexa vaccinees (95\% CI $2 \cdot 4-8 \cdot 9$ ). These findings suggest that short-term hepatitis B immunogenicity in Hexavac vaccinees may also be weaker. Further studies are warranted to assess whether Hexavac vaccinees should be re-vaccinated or receive a booster vaccination before these birth cohorts reach adolescence.

Key words: Hepatitis B, public health, vaccination (immunization), vaccines.

\section{INTRODUCTION}

Vaccination against hepatitis B in early childhood is the most effective way of preventing spread of infection. According to published data, available vaccines induce a protective antibody response to hepatitis B surface antigen (anti-HBs) in about $95 \%$ of vaccinees and confer long-lasting protection against hepatitis $\mathrm{B}$ infection in immunocompetent individuals [1-8]. The magnitude of the anti-HBs response is largely

\footnotetext{
* Author for correspondence: Dr D. Radun, M.Sc., Department for Infectious Disease Epidemiology, Robert Koch Institute, DGZRing 1, 13086 Berlin, Germany.

(Email: RadunD@rki.de)
}

influenced by vaccine regimen, body mass, site and route of injection, immunosuppression, and age [9-12]. A peak anti-HBs concentration $\geqslant 10$ milli international units per millilitre $(\mathrm{mIU} / \mathrm{ml})$ measured 1 month after completion of primary vaccination course is considered to provide long-term immunity [5]. Even in individuals whose anti-HBs concentration decreases to lower or undetectable levels, protection against disease is likely to persist due to the maintenance of $\mathrm{HBsAg}$ specific immunological memory [13-16]. Nonetheless, it has not been firmly established whether booster vaccination is necessary and if so, at what intervals $[1-3,6,8,17]$.

Germany, a country with a low endemicity for hepatitis $\mathrm{B}$, introduced national recommendations for 
universal hepatitis B vaccination of infants, children, and adolescents in 1995. In 2000, two hexavalent vaccines, Hexavac ${ }^{\circledR}$ (Sanofi Pasteur MSD), France and Infanrix hexa ${ }^{\circledR}$ (GlaxoSmithKline, Belgium), against diphtheria, tetanus, pertussis, hepatitis B, polio and Haemophilus influenzae type $\mathrm{b}$ were approved for use in Germany. Both vaccines were licensed for either a three- or a four-dose primary series. In Germany the routine vaccination schedule for hexavalent vaccines recommends four doses at ages of 2, 3, 4, and 11-14 months, with at least 6 months between the third and fourth dose [18]. The recommendations made no preference for either vaccine.

Hepatitis B vaccination coverage in Germany increased markedly in conjunction with the availability of hexavalent vaccines and data from the German National Health Interview and Examination Survey for Children and Adolescents (KiGGS) shows that hexavalent vaccines practically replaced vaccination with monovalent hepatitis B vaccinees during childhood [19, 20].

In 2005, the European Medicines Agency (EMEA) suspended the marketing authorization for Hexavac due to precautionary concerns about the long-term persistence of immunogenicity of the hepatitis B component of the vaccine [21, 22]. Additionally, there was some evidence that Hexavac-vaccinated children who achieved anti-HBs levels between 10 and $100 \mathrm{mIU} / \mathrm{ml}$ post-primary vaccination had no or a lower response to booster vaccinations with monovalent hepatitis B vaccines given at age 7-9 years, compared to children whose initial levels were $>100 \mathrm{mIU} / \mathrm{ml}$ [22]. Hexavac was licensed in Germany in late October 2000 and between 2001 and the time of its suspension in September 2005, 5.8 million doses of vaccine were prescribed, corresponding to at least 1.5 million vaccinated children (IMS Health, written communication). Hexavac was also licensed in nine other European countries as well as in 19 additional countries worldwide [21].

Current knowledge on the immunogenicity of Hexavac and Infanrix hexa has largely been gained from clinical trials [23-27]. Questions remain about the short- and long-term hepatitis B immunogenicity in children vaccinated with these hexavalent vaccines during routine immunization. To investigate this, we compared anti-HBs levels in children in Germany fully vaccinated with either Hexavac or Infanrix hexa between 2000 and 2005 using data from a large cross-sectional survey. We also assessed factors associated with anti-HBs concentrations $<10 \mathrm{mIU} / \mathrm{ml}$.

\section{METHODS}

\section{The German Health Survey for Children and Adolescents (KiGGS)}

A nationwide cross-sectional survey, KiGGS, was conducted between 2003 and 2006 [28]. KiGGS was based on a nationally representative sample of children and adolescents aged $0-17$ years with main residence in Germany. Participants were enrolled in two steps: first, 167 study locations (sample points) were chosen; second, subjects were randomly selected from the official resident registers in the 167 sample locations. A total of 17641 subjects (8656 girls, 8985 boys) participated in the survey (response 66.6\%). Analysis of the non-responder questionnaires showed that the collected data gave comprehensive and nationally representative evidence on the health status of children and adolescents. To ensure that estimates derived from KiGGS were representative at the national level, weights were applied in statistical analyses to correct for regional differences in the distribution of age structure, sex, and nationality, and to compensate for oversampling of individuals from Eastern Germany which was undertaken to achieve sufficient statistical power.

Data on age, sex, anthropometric variables, chronic diseases, socio-economic status, and a large number of health indicators were collected through interviews and self-completed questionnaires. A wide range of blood and urine tests were performed. Vaccination status was extracted from hand-held vaccination cards. A detailed description of the sampling method has been published elsewhere [28, 29].

\section{Hepatitis B testing}

Laboratory tests for anti-HBs and antibodies to hepatitis B core antigen (anti-HBc) were performed in the majority of children aged 3-17 years $(88 \%)$. However, the proportion of children tested for anti$\mathrm{HBs}$ and anti-HBc was smaller in the younger age groups due to more frequent refusals or blood sampling failures. Anti-HBs and anti-HBc were determined by electrochemiluminescence immunoassay (ECLIA, Roche Diagnostics, Switzerland) using automatic ELECSYS 2010 Analyzer. Only samples which were anti-HBc positive were tested for hepatitis B surface antigen (HBsAg). Anti-HBs concentration was determined quantitatively in the range $2-1000 \mathrm{mIU} /$ $\mathrm{ml}$. Levels $<2 \mathrm{mIU} / \mathrm{ml}$ were classified as undetectable, whereas concentrations $>1000 \mathrm{mIU} / \mathrm{ml}$ were not 
further diluted. Tests for anti-HBc and HBsAg were qualitative. Details of the laboratory methods have previously been published [30].

\section{Selection of subjects vaccinated with hexavalent vaccines - subgroup analysis}

For the purpose of this study, we selected a subset of children from KiGGS. We included only children who fulfilled the following four criteria: (i) previous vaccination either with only four doses of Hexavac or only four doses of Infanrix hexa; (ii) complete and original vaccination card available; (iii) at least 5 months between the third and fourth vaccination dose, and (iv) results of anti-HBs and anti-HBc testing available. Children who were positive for anti-HBc or HBsAg, or who had been passively or actively immunized at birth were excluded from our dataset. For the selected subgroup we extracted the following from the KiGGS database: data on age, sex, birth weight, results of anti-HBs testing, brand and dates of hexavalent vaccination, information on diseases which may impair immunity, and concomitant administration of other available childhood vaccines [measles, mumps and rubella (MMR), pneumococcal, meningococcal, varicella, influenza, hepatitis $\mathrm{A}$, and tick-borne encephalitis].

\section{Statistical analysis}

We grouped children according to anti-HBs levels $(<2$, 2 to $<10,10$ to $<100,100$ to $<1000, \geqslant 1000 \mathrm{mIU} / \mathrm{ml}$ ) and assessed differences in anti-HBs between Hexavac and Infanrix hexa vaccinees using Pearson's $\chi^{2}$ test.

We performed weighted bivariate and multivariable logistic regression analyses to identify factors associated with anti-HBs concentration $<10 \mathrm{mIU} / \mathrm{ml}$. The weighted analyses were performed applying the weights developed for KiGGS as described above. For these analyses, we grouped subjects by vaccine (four doses of Hexavac $v$ s. four doses of Infanrix hexa), anti-HBs concentration $(<10 \mathrm{mIU} / \mathrm{ml}$ vs. $\geqslant 10 \mathrm{mIU} /$ $\mathrm{ml}$ ), sex (male vs. female), birth weight $[<2499 \mathrm{~g}$ (underweight), $\geqslant 4500 \mathrm{~g}$ (overweight), $\geqslant 2500-4499 \mathrm{~g}$ (normal)], concomitantly vaccinated [hexavalent vaccine administered alone vs. hexavalent vaccine administered with other vaccine(s)], and age at first vaccination dose ( $\geqslant 6$ months or $<6$ months). We included bivariate predictors with $P<0.25$ in the multivariable model. Using a backward stepwise elimination $(P>0.05)$ in the multivariable analysis, we removed variables not associated with anti-HBs
Table 1. Demographic, vaccination, and birth weight characteristics (weighted) of study participants by vaccine received

\begin{tabular}{lll}
\hline \hline Characteristic & $\begin{array}{l}\text { Infanrix hexa }^{\circledR} \\
(n=227)\end{array}$ & $\begin{array}{l}\operatorname{Hexavac}^{\circledR} \\
(n=250)\end{array}$ \\
\hline $\begin{array}{l}\text { Female } \\
\begin{array}{l}\text { Mean age (years) } \\
(\text { min-max) }\end{array}\end{array}$ & $\begin{array}{c}49 \cdot 7 \% \\
3 \cdot 44(3 \cdot 0-6 \cdot 0)\end{array}$ & $\begin{array}{l}49 \cdot 0 \% \\
3 \cdot 58(3 \cdot 0-5 \cdot 5)\end{array}$ \\
$\begin{array}{l}\text { Mean time since } \\
\text { last vaccination } \\
\text { (years) (min-max) }\end{array}$ & $2 \cdot 40(0 \cdot 35-4 \cdot 45)$ & $2 \cdot 49(0 \cdot 31-4 \cdot 37)$ \\
$\begin{array}{l}\text { Mean birth } \\
\text { weight }(\mathrm{kg})\end{array}$ & $3 \cdot 40(1 \cdot 88-5 \cdot 27)^{*}$ & $3 \cdot 39(0 \cdot 68-4 \cdot 56) \dagger$ \\
$($ min-max $)$ & & \\
\hline \hline
\end{tabular}

$* n=222$.

$\dagger n=246$.

$<10 \mathrm{mIU} / \mathrm{ml}$. The prevalence of children with antiHBs $<10 \mathrm{mIU} / \mathrm{ml}$ by time elapsed since completed vaccination was estimated for each vaccine group using multivariable logistic regression analysis. All possible interaction terms were tested separately. $P$ values $<0.05$ were considered statistically significant. All analyses were performed with Stata 9.0 (StataCorp, USA).

\section{RESULTS}

\section{Study population}

A total of 477 children fulfilled the inclusion criteria; 227 were vaccinated with Infanrix hexa, and 250 with Hexavac. The characteristics of the two vaccine groups were similar in terms of sex, age, time since completion of vaccination and birth weight (Table 1). The only vaccines administered concomitantly with Hexavac or Infanrix hexa were MMR (Hexavac, $n=12$; Infanrix hexa, $n=16$ ) and pneumococcal vaccine (Hexavac, $n=6$; Infanrix hexa, $n=1$ ).

No child was known to have a disease associated with impaired immunity, e.g. nephrotic syndrome, Down's syndrome, or human immunodeficiency virus infection.

We excluded 201 children who had received four doses of Hexavac or Infanrix hexa but did not fulfil the other inclusion criteria. Of these, 183 had not been tested for anti-HBs, 16 had received the fourth dose $<5$ months after the third dose, and two were positive for anti-HBc.

Excluded children were younger (median age 3.69 years) than those fulfilling all inclusion criteria (median 
Table 2. Anti-HBs levels in Infanrix hexa ${ }^{\circledR}$ and Hexavac ${ }^{\circledR}$ vaccinees 0-3-4.5 years after fourth vaccine dose

Prevalence $(\%)$ of anti-HBs $(\mathrm{mIU} / \mathrm{ml})$ with binomial $95 \% \mathrm{CI}$

\begin{tabular}{|c|c|c|c|c|c|}
\hline Vaccine & Undetectable & 2 to $<10$ & 10 to $<100$ & 100 to $<1000$ & $\geqslant 1000$ \\
\hline $\begin{array}{l}\text { Infanrix hexa } \\
(n=227)\end{array}$ & $2 \cdot 0(0 \cdot 8-4 \cdot 5)$ & $2 \cdot 7(1 \cdot 1-6 \cdot 5)$ & $17 \cdot 7(12 \cdot 8-24 \cdot 0)$ & $36 \cdot 1(28 \cdot 3-44 \cdot 8)$ & $41 \cdot 5(33 \cdot 8-49 \cdot 7)$ \\
\hline $\begin{array}{l}\text { Hexavac } \\
(n=250)\end{array}$ & $12 \cdot 9(8 \cdot 9-18 \cdot 3)$ & $12 \cdot 4(8 \cdot 4-17 \cdot 9)$ & $37 \cdot 9(31 \cdot 1-45 \cdot 3)$ & $29 \cdot 7(23 \cdot 4-36 \cdot 8)$ & $7 \cdot 1(4 \cdot 2-11 \cdot 8)$ \\
\hline Pearson's $\chi^{2}$ & \multicolumn{5}{|c|}{$\left(\chi^{2}=106 \cdot 718, P<0 \cdot 001\right)$} \\
\hline
\end{tabular}

age 3.90 years, Wilcoxon rank sum test $2.53 ; P=$ $0 \cdot 011)$.

\section{Prevalence of protective anti-HBs}

The distribution of anti-HBs levels differed significantly between the two vaccine groups $0 \cdot 3-4 \cdot 5$ years after the fourth dose (Table 2). For instance, in children vaccinated with Hexavac, $25 \cdot 3 \% \quad(95 \%$ CI 19.0-32.8) had anti-HBs $<10 \mathrm{mIU} / \mathrm{ml}$ compared to $4 \cdot 7 \%$ (95\% CI $2 \cdot 4-8.9)$ of children vaccinated with Infanrix hexa. A significantly larger proportion of children vaccinated with Infanrix hexa $(41.5 \%)$ had very high anti-HBs levels $(\geqslant 1000 \mathrm{mIU} / \mathrm{ml})$ compared with Hexavac vaccinees $(7 \cdot 1 \%)$. This difference was also pronounced in children who had completed their vaccination $\leqslant 2$ years previously $(n=126)$. In this group, $66 \cdot 2 \%$ of Infanrix hexa vaccinees had an anti-HBs level $\geqslant 1000 \mathrm{mIU} / \mathrm{ml}$ compared to $11.9 \%$ of Hexavac vaccinees (Pearson's $\chi^{2}=36 \cdot 3, P<0 \cdot 001$ ).

\section{Bivariate logistic regression analysis}

In the weighted bivariate logistic regression analysis, vaccine type, birth weight, and years since completion of vaccination were significantly associated with an anti-HBs level $<10 \mathrm{mIU} / \mathrm{ml}(P<0 \cdot 01$, Table 3$)$, while there was no association with sex, age at first vaccination dose, and concomitant vaccinations $(P>0 \cdot 25)$.

\section{Multivariable logistic regression analysis}

In the multivariable logistic regression analysis, vaccine type, birth weight, and years since last vaccination remained as independent risk factors for anti-HBs $<10 \mathrm{mIU} / \mathrm{ml}$ (Table 4). None of the interaction terms significantly improved the model fit and were thus not considered in the final model. The odds of having an anti-HBs concentration $<10 \mathrm{mIU} / \mathrm{ml}$ was eight times higher in children vaccinated with Hexavac compared to children vaccinated with Infanrix hexa and increased by 1.8 for every additional year since completion of vaccination. Being underweight at birth was also strongly associated with anti-HBs levels $<10 \mathrm{mIU} / \mathrm{ml}$.

\section{Persistence of anti-HBs by time since completed vaccination}

The prevalence of individuals with anti-HBs $<10 \mathrm{mIU} / \mathrm{ml}$ was significantly higher at any point in time since vaccination in Hexavac vaccinees than in Infanrix hexa vaccinees as modelled using logistic regression (Fig. 1). For example 1 year post-vaccination, the estimated prevalence of anti-HBs $<10 \mathrm{mIU} / \mathrm{ml}$ was $13.5 \%(95 \%$ CI $7 \cdot 6-22.9)$ in children vaccinated with Hexavac vs. $2 \cdot 3 \%(95 \%$ CI $0 \cdot 8-6 \cdot 1)$ in children vaccinated with Infanrix hexa. For children who completed vaccination 4 years earlier, an estimated $40.7 \%$ (95\% CI 25.9-57.3) of Hexavac vaccinees had anti$\mathrm{HBs}<10 \mathrm{mIU} / \mathrm{ml}$ compared to $9 \cdot 2 \% \quad(95 \% \mathrm{CI}$ 4.5-17.9) of Infanrix hexa vaccinees (Fig. 1).

\section{DISCUSSION}

This study comparing anti-HBs levels in children vaccinated only with Hexavac to those in children vaccinated only with Infanrix hexa provides further evidence on the immunogenicity of the hepatitis B component of Hexavac. The significantly higher proportion of children in our analysis with anti-HBs levels both undetectable (12.9\%) and $<10 \mathrm{mIU} / \mathrm{ml}$ $(25.3 \%)$ in those vaccinated with Hexavac compared to those vaccinated with Infanrix hexa $(2.0 \%$ and $4 \cdot 7 \%$, respectively) suggests that short-term as well as long-term immunogenicity in Hexavac vaccinees may be weaker.

Several studies have shown that the persistence of measurable antibody levels is related to peak 
Table 3. Results of weighted bivariate analysis of potential risk factors for anti-HBs $<10 \mathrm{mIU} / \mathrm{ml}$

\begin{tabular}{|c|c|c|c|c|}
\hline Variable & $\begin{array}{l}\text { Percent with anti-HBs } \\
<10 \mathrm{mIU} / \mathrm{ml}\end{array}$ & POR* & $95 \% \mathrm{CI} \dagger$ & $P$ value \\
\hline \multicolumn{5}{|l|}{$\operatorname{Sex}(n=477)$} \\
\hline Male $(n=239)$ & $13 \cdot 9$ & $1 \cdot 00$ & & \\
\hline Female $(n=238)$ & $17 \cdot 7$ & $1 \cdot 34$ & $0 \cdot 72-2 \cdot 49$ & $0 \cdot 349$ \\
\hline \multicolumn{5}{|l|}{ Vaccine $(n=477)$} \\
\hline Infanrix hexa ${ }^{\mathbb{R}}(n=227)$ & $4 \cdot 7$ & $1 \cdot 00$ & & \\
\hline $\operatorname{Hexavac}^{\mathbb{R}}(n=250)$ & $25 \cdot 3$ & $6 \cdot 94$ & $3 \cdot 28-14 \cdot 67$ & $<0 \cdot 001$ \\
\hline \multicolumn{5}{|l|}{ Birth weight $\$(n=461)$} \\
\hline Normal weight $(n=433)$ & $14 \cdot 2$ & $1 \cdot 00$ & & \\
\hline Underweight $(n=28)$ & $49 \cdot 8$ & $5 \cdot 98$ & $2 \cdot 18-16 \cdot 4$ & $<0 \cdot 001$ \\
\hline \multicolumn{5}{|l|}{ Age first dose $(n=477)$} \\
\hline$\geqslant 6$ months $(n=23)$ & $13 \cdot 3$ & $1 \cdot 00$ & & \\
\hline$<6$ months $(n=454)$ & $15 \cdot 9$ & $1 \cdot 23$ & $0 \cdot 34-4 \cdot 42$ & $0 \cdot 750$ \\
\hline \multicolumn{5}{|l|}{ Concomitant MMR vaccination $(n=477)$} \\
\hline No $(n=449)$ & $17 \cdot 2$ & $1 \cdot 00$ & & \\
\hline Yes $(n=26)$ & $14 \cdot 3$ & $1 \cdot 00$ & $0 \cdot 25-3 \cdot 99$ & 0.997 \\
\hline \multicolumn{5}{|c|}{ Concomitant pneumococcal vaccination $(n=477)$} \\
\hline No $(n=470)$ & & $1 \cdot 0$ & & \\
\hline Yes $(n=7)$ & $28 \cdot 6$ & $1 \cdot 74$ & $0 \cdot 29-10 \cdot 48$ & $0 \cdot 540$ \\
\hline Years since last vaccination $(n=477)$ & & 1.69 & $1 \cdot 17-2 \cdot 43$ & $0 \cdot 005$ \\
\hline
\end{tabular}

MMR, Measles, mumps and rubella.

* Prevalence odds ratio.

$\dagger$ Binomial $95 \%$ confidence intervals.

$\$$ Normal weight : $2500-4499 \mathrm{~g}$, underweight : $\leqslant 2499 \mathrm{~g}$ (according to ICD-10 [48]). Data on birth weight was missing for nine children. Seven children classified as overweight could not be included in the analysis as they all had anti-HBs $>10 \mathrm{mIU} / \mathrm{ml}$. $\S$ Twenty-two were born preterm.

Table 4. Results of the weighted multivariable logistic regression analysis, showing factors associated with anti-HBs $<10 \mathrm{mIU} / \mathrm{ml}(n=461)$

\begin{tabular}{|c|c|c|c|c|}
\hline Variable & $\begin{array}{l}\text { Proportion (\%) with } \\
\text { anti-HBs }<10 \mathrm{mIU} / \mathrm{ml}\end{array}$ & Adj. POR* & $95 \% \mathrm{CI} \dagger$ & $P$ value \\
\hline Infanrix hexa ${ }^{\circledR}(n=218)$ & $4 \cdot 7$ & 1.00 & & \\
\hline $\operatorname{Hexavac}^{\mathbb{R}}(n=243)$ & $25 \cdot 3$ & $8 \cdot 22$ & $3 \cdot 73-18 \cdot 1$ & $<0 \cdot 001$ \\
\hline Normal weight $(n=433)$ & $14 \cdot 2$ & $1 \cdot 00$ & & \\
\hline Underweight $(n=28)$ & $49 \cdot 8$ & $10 \cdot 35$ & $3 \cdot 45-31 \cdot 0$ & $<0 \cdot 001$ \\
\hline Years since completed vaccination & - & $1 \cdot 77$ & $1 \cdot 21-2 \cdot 58$ & $0 \cdot 003$ \\
\hline
\end{tabular}

* Adjusted prevalence odds ratio.

$\dagger$ Binominal $95 \%$ confidence intervals.

anti-HBs levels after immunization [31-33]. The increasingly higher prevalence of anti-HBs $<10 \mathrm{mIU} / \mathrm{ml}$ with time since completed vaccination in Hexavac vaccinees compared to Infanrix hexa vaccinees as suggested by our model is consistent with lower reported anti-HBs levels post-primary vaccination for Hexavac [27]. Similarly, the significantly higher proportion of children vaccinated with Infanrix hexa having anti-HBs levels $\geqslant 1000 \mathrm{mIU} / \mathrm{ml}$ within 2 years of the last vaccination compared to those vaccinated with Hexavac implies that Infanrix hexa vaccinees might have responded with substantially higher antiHBs levels post-vaccination.

According to EMEA, the lower anti-HBs levels in Hexavac vaccinees compared to Infanrix hexa vaccinees may be attributable to variability in the 


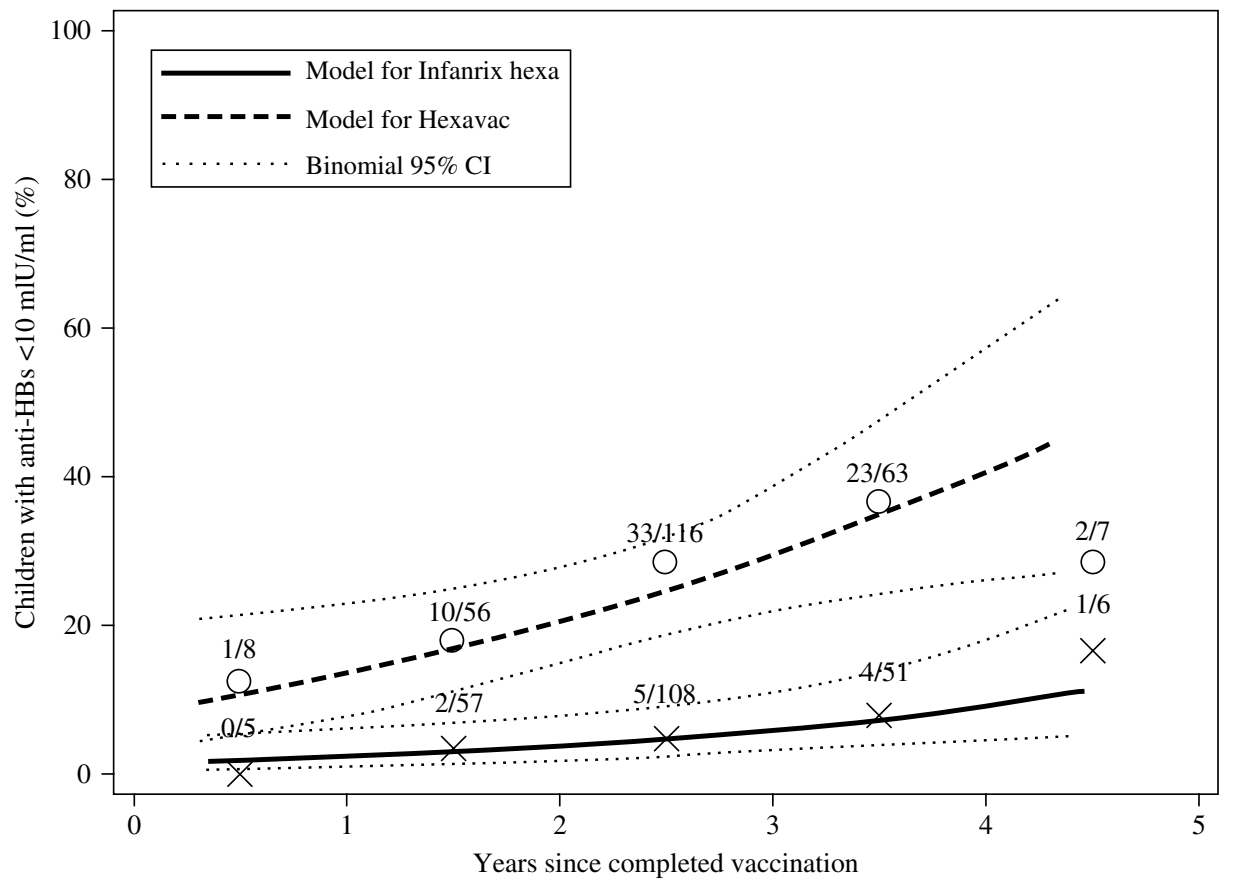

Fig. 1. Estimated prevalence of Hexavac ${ }^{\mathbb{R}}$ and Infanrix hexa ${ }^{\mathbb{R}}$ vaccinees with anti-HBs $<10 \mathrm{mIU} / \mathrm{ml}$ according to time since completed vaccination as modelled by weighted logistic regression analysis. Open circles (Hexavac) and crosses (Infanrix hexa) show the number of subjects with anti-HBs levels $<10 \mathrm{mIU} / \mathrm{ml}$ in all subjects within each successive year after completed vaccination.

production process of the hepatitis $\mathrm{B}$ component of Hexavac [22]. The lower recombinant HBsAg content in the Hexavac vaccine $(5 \mu \mathrm{g} H B s A g)$ compared to that of the Infanrix hexa vaccine $(10 \mu \mathrm{g}$ HBsAg) may also play a role; however, an Italian study showed that a high proportion of children $(92.9 \%)$ vaccinated in infancy with either $5 \mu \mathrm{g}$ or $10 \mu \mathrm{g}$ HBsAg had antiHBs levels $\geqslant 10 \mathrm{mIU} / 15$ years after vaccination [34]. In that study, simultaneous vaccination with combined diphtheria and tetanus vaccine or with polio vaccine was associated with lower anti-HBs levels. To what extent other differences in hexavalent vaccine composition - particularly differences in the concentration of the adjuvant aluminium hydroxide - might be relevant, is not known. As the children in the two vaccine groups were very similar in terms of a number of factors which might influence response to vaccination, the observed anti-HBs differences in our study are unlikely to be due to differences between the comparison groups.

The findings presented here are consistent with previous reports. In particular, another recent Italian study in children attending local health units found that $31 \%$ of 113 Hexavac vaccinees had anti-HBs $<10 \mathrm{mIU} / \mathrm{ml}$ compared to only $4 \%$ of 129 Infanrix hexa vaccinees 15 months post-primary vaccination with three doses [35]. A clinical trial showed that both the proportion of children with anti-HBs $\geqslant 10 \mathrm{mIU} / \mathrm{ml}$ as well as geometric mean titres (GMTs) were significantly higher in children vaccinated with three doses of Infanrix hexa compared to three doses of Hexavac 1 month post-vaccination [27]. A follow-up trial reported significantly higher anti-HBs GMTs as well as seroprevalence rates at age 11-23 months in infants primed with Infanrix hexa in the first study [26]. However, following a fourth (booster) dose, the proportion of children with antiHBs $\geqslant 10 \mathrm{mIU} / \mathrm{ml}$ was similar in the two groups, although GMTs remained significantly higher in Infanrix hexa vaccinees. Finally, a vaccine trial in Germany showed that $>90 \%$ of children vaccinated with four doses of Infanrix hexa had persistent protective anti-HBs levels 3.5-4 years after administration of a fourth dose, thus supporting the findings presented here [36].

Low birth weight was also strongly and independently associated with anti-HBs levels $<10 \mathrm{mIU} / \mathrm{ml}$ in this study. Since a high proportion $(79 \%)$ of the children with low birth weight $(n=28)$ were born preterm, these findings are in keeping with other studies which have shown decreased response to hepatitis B vaccines in preterm infants [37-39]. 
We found no association between anti-HBs levels $<10 \mathrm{mIU} / \mathrm{ml}$ and concomitant vaccination with MMR or pneumococcal vaccine; however, the number of concomitantly vaccinated children was small. Previous studies have reported lower geometric mean anti-HBs levels with concomitant administration of hexavalent vaccines and pneumococcal conjugate vaccine $[40,41]$.

Our study has two major limitations. The first limitation is inherent in the design of a cross-sectional survey. Because data were only collected at a single point in time for each participant, we could not assess individual anti-HBs levels over time. Thus, we could not firmly establish whether undetectable anti-HBs levels were mainly due to primary vaccine failure, i.e. non-response, or secondary vaccine failure, i.e. waning immunity after seroconversion. Second, data on several factors which might influence the response to vaccination, including non-chronic diseases, medication (e.g. steroids), body weight at the time of vaccination and vaccine administration route were unavailable in this secondary data analysis. However, it appears unlikely that children who received Hexavac would differ from children vaccinated with Infanrix hexa in these aspects.

EMEA's suspension of Hexavac in 2005 was considered a precautionary measure and immediate revaccination of children who completed primary vaccination with Hexavac was not deemed necessary [21]. However, the degree of short- and long-term protection against hepatitis B infection in children vaccinated with Hexavac remains unclear. Overall, the clinical significance of anti-HBs $<10 \mathrm{mIU} / \mathrm{ml}$ in immunized individuals is not well understood. Studies suggest that long-term persistence of high anti-HBs levels, as well as immunological memory and the degree of protection from infection are associated with peak anti-HBs concentrations after vaccination [31, 33, 42-44]. Several studies have shown that healthy individuals with an anti-HBs concentration $<10 \mathrm{mIU} / \mathrm{ml}$ can be infected with hepatitis $\mathrm{B}$, but mostly retain protection against chronic infection provided that they initially responded well to the vaccine [2, 3, 17, 33, 42, 43].

Most studies investigating long-term immunogenicity of hepatitis B vaccines and related immunological memory followed up relatively small numbers of vaccinees in settings with intermediate or high hepatitis B endemicity [5, 33, 42-45]. Some or all subjects in these studies were either vaccinated with lower [43-45] or higher $[4,33]$ doses of $\mathrm{HBsAg-containing} \mathrm{vaccines,}$ were vaccinated at birth [5, 43-45], or were vaccinated with plasma-derived vaccines [33, 45]. Therefore, results of these studies are not directly applicable to the German setting. However, some of these studies showed a decrease in anti-HBs concentrations to $<10 \mathrm{mIU} / \mathrm{ml}$ over a period of up to 15 years in up to $60 \%$ of vaccinees $[5,33,43,45]$, with up to $50 \%$ unable to mount an anamnestic immune response $[5,43$, 45]. Conversely, an Italian study found that $91 \cdot 2 \%$ of adolescents vaccinated with three doses of recombinant vaccine containing a high dose of $\mathrm{HBsAg}$ $(20 \mu \mathrm{g})$ retained anti-HBs-levels of $>10 \mathrm{mIU} / \mathrm{ml}$ more than 10 years later [4].

According to EMEA, there is some degree of uncertainty as to whether Hexavac vaccinees with an initial immune response of $10-100 \mathrm{mIU} / \mathrm{ml}$ anti-HBs after primary vaccination can mount an anamnestic response to a booster dose with monovalent hepatitis B vaccines [22]. A study by Giambi et al. showed that $2 / 25(8 \%)$ Hexavac vaccinees vs. $0 / 5(0 \%)$ Infanrix hexa vaccinees with anti-HBs levels $<10 \mathrm{mIU} / \mathrm{ml} 15$ months after receipt of three primary doses did not attain anti-HBs levels $>10 \mathrm{mIU} / \mathrm{ml} 1$ month after a booster dose with a monovalent hepatitis $\mathrm{B}$ vaccine; however, the difference was not statistically significant [35].

Since hepatitis B booster vaccination is currently not recommended for the general population in Germany [18], the success of the childhood immunization policy relies on long-term immunity into adolescence and beyond, when the risk of hepatitis B infection increases with onset of sexual activity [46]. In countries with low endemicity, where natural exposure to hepatitis B is limited, booster vaccinations may be necessary to sustain immunity.

To establish whether Hexavac-vaccinated children might require re-vaccination or a booster dose, studies should be conducted to assess the ability of children with low anti-HBs levels after completion of the primary series to mount an anamnestic response to booster vaccination. Additional studies to assess the long-term persistence of anti-HBs and investigation of possible breakthrough infections in high-risk populations should also be performed.

In Switzerland, administration of one dose of a monovalent hepatitis $\mathrm{B}$ vaccine before the age of 8 years to children who had received four doses of Hexavac was recommended following the suspension of the vaccine [47]. The decision to implement a similar strategy in Germany would largely depend on the results of the above-mentioned studies. 


\section{DECLARATION OF INTEREST}

The authors were employees at the Robert Koch Institute (P.J., C.P.M., W.H., W.T., C.M., M.H., M.S., D.R.) and University Regensburg (W.J.) at the time of the study. All authors, the Robert Koch Institute, and University Regensburg, report no financial or other conflict of interest.

\section{REFERENCES}

1. Floreani A, et al. Long-term persistence of anti-HBs after vaccination against $\mathrm{HBV}$ : an 18 year experience in health care workers. Vaccine 2004; 22: 608-611.

2. European Consensus Group on Hepatitis B Immunity. Are booster immunisations needed for lifelong hepatitis B immunity? Lancet $2000 ; 12$ : 561-565.

3. FitzSimons D, et al. Long-term efficacy of hepatitis B vaccine, booster policy, and impact of hepatitis B virus mutants. Vaccine 2005; 23: 4158-4166.

4. Gabbuti A, et al. Long-term immunogenicity of hepatitis B vaccination in a cohort of Italian healthy adolescents. Vaccine 2007; 25: 3129-3132.

5. Hammitt LL, et al. Hepatitis B immunity in children vaccinated with recombinant hepatitis B vaccine beginning at birth: A follow-up study at 15 years. Vaccine 2007; 25 : 6958-6964.

6. Lin YC, et al. Long-term immunogenicity and efficacy of universal hepatitis B virus vaccination in Taiwan. Journal of Infectious Diseases 2003; 187: 134-138.

7. Watson B, et al. Persistence of immunologic memory for 13 years in recipients of a recombinant hepatitis B vaccine. Vaccine 2001; 19: 3164-3168.

8. Zanetti A, et al. Long-term immunogenicity of hepatitis B vaccination and policy for booster: an Italian multicentre study. Lancet 2005; 366: 1379-1384.

9. Shaw FE, et al. Effect of anatomic injection site, age and smoking on the immune response to hepatitis $\mathrm{B}$ vaccination. Vaccine 1989; 7: 425-430.

10. Bryan JP, et al. Low-dose intradermal and intramuscular vaccination against hepatitis B. Clinical Infectious Diseases 1992; 14: 697-707.

11. Weber DJ, et al. Obesity as a predictor of poor antibody response to hepatitis B plasma. Journal of the American Medical Association 1985; 254: 3187-3189.

12. Zuckerman JN. Nonresponse to hepatitis B vaccines and the kinetics of Anti-HBs production. Journal of Medical Virology 1996; 50: 283-288.

13. Dentinger $\mathbf{C}$, et al. Persistence of antibody to hepatitis B and protection from disease among Alaska natives immunized at birth. Pediatric Infectious Disease Journal 2005; 24 : 786-792.

14. Koff RS. Immunogenicity of hepatitis B vaccines: implications of immune memory. Vaccine 2002; 20: 3695-3701.

15. Wang R, et al. Long-term persistence of $\mathrm{T}$ cell memory to HBsAg after hepatitis B vaccination. World Journal of Gastroenterology 2004; 10 : 260-263.
16. Bauer T, Jilg W. Hepatitis B surface antigen-specific T and $\mathrm{B}$ cell memory in individuals who had lost protective antibodies after hepatitis $\mathrm{B}$ vaccination. Vaccine 2006; 24 : 572-577.

17. Banatvala JE, Van Damme P. Hepatitis B vaccine - do we need boosters? Journal of Viral Hepatitis 2003; 10: $1-6$.

18. Ständige Impfkommission am Robert Koch-Institut. Recommendations of the German Standing Vaccination Committee (STIKO) at the Robert Koch Institute (as of July 2007) [in German]. Epidemiologisches Bulletin 2007; 30: 267-286.

19. Poethko-Müller C, Kuhnert R, Schlaud M. Vaccination coverage and predictors for vaccination level. Results of the German Health Interview and Examination Survey for Children and Adolescents (KiGGS). Bundesgesundheitsblatt Gesundheitsforschung Gesundheitsschutz 2007; 50: 851-862.

20. Kalies H, et al. Immunisation status of children in Germany: temporal trends and regional differences. European Journal of Pediatrics 2006; 165: 30-36.

21. European Medicines Agency. Questions and answers on the suspension of Hexavac, 2005, London, United Kingdom (http://www.ema.europa.eu/humandocs/PDFs/ EPAR/Hexavac/30488805en.pdf). Accessed 26 June 2009.

22. European Medicines Agency. Scientific conclusions and grounds for the suspension of the marketing authorisation of Hexavac presented by the EMEA, 2005 (http://www.emea.europa.eu/humandocs/PDFs/EPAR/ Hexavac/Hexavac-H-298-Z-28-en.pdf). Accessed 26 June 2009.

23. Kilpi T, et al. Immunogenicity and reactogenicity of two diphtheria-tetanus-acellular pertussis-hepatitis B-inactivated polio virus-Haemophilus influenzae type $b$ vaccines administered at 3, 5 and 11-12 months of age. Human Vaccines 2009; 5: 18-25.

24. Mallet E, et al. Immunogenicity and safety of a new liquid hexavalent combined vaccine compared with separate administration of reference licensed vaccines in infants. Pediatric Infectious Disease Journal 2000; 19: 1119-1127.

25. Mallet E, et al. A liquid hexavalent combined vaccine against diphtheria, tetanus, pertussis, poliomyelitis, Haemophilus influenzae type B and hepatitis B: review of immunogenicity and safety. Vaccine 2004; 22: 1343 1357.

26. Tichmann I, et al. Persistence of antibodies in children primed with two different hexavalent diphtheria, tetanus, acellular pertussis, hepatitis B, inactivated poliovirus and Haemophilus influenzae type $b$ vaccines and evaluation of booster vaccination. Human Vaccines 2006; 2 : 249-254.

27. Tichmann I, et al. Comparison of the immunogenicity and reactogenicity of two commercially available hexavalent vaccines administered as a primary vaccination course at 2, 4 and 6 months of age. Vaccine 2005; 23: 3272-3279.

28. Kurth B-M, et al. The challenge of comprehensively mapping children's health in a nation-wide health 
survey: design of the German KiGGS Study. $B M C$ Public Health 2008; 8: 196.

29. Hölling H, et al. The German Health Interview and Examination Survey for Children and Adolescents (KiGGS): study management and conduct of fieldwork [in German]. Bundesgesundheitsblatt Gesundheitsforschung Gesundheitsschutz 2007; 50: 557-566.

30. Thierfelder W, et al. Biochemical measures in the German Health Interview and Examination Survey for Children and Adolescents (KiGGS). Bundesgesundheitsblatt Gesundheitsforschung Gesundheitsschutz 2007; 50: 757-770.

31. Jilg W, Schmidt M, Deinhardt F. Persistence of specific antibodies after hepatitis B vaccination. Journal of Hepatology 1988; 6: 201-207.

32. van der Sande MA, et al. Long-term protection against carriage of hepatitis B virus after infant vaccination. Journal of Infectious Diseases 2006; 193: 1528-1535.

33. McMahon BJ, et al. Antibody levels and protection after hepatitis B vaccination: results of a 15-year follow-up. Annals of Internal Medicine 2005; 142: 333-341.

34. Faustini A, et al. Persistence of anti-HBs 5 years after the introduction of routine infant and adolescent vaccination in Italy. Vaccine 2001 ; 19: 2812-2818.

35. Giambi C, et al. A cohort study to evaluate persistence of hepatitis B immunogenicity after administration of hexavalent vaccines. BMC Infectious Diseases 2008; 8: 100.

36. Heininger $\mathbf{U}$, et al. Booster immunization with a hexavalent diphtheria, tetanus, acellular pertussis, hepatitis $\mathrm{B}$, inactivated poliovirus vaccine and Haemophilus influenzae type $b$ conjugate combination vaccine in the second year of life: safety, immunogenicity and persistence of antibody responses. Vaccine 2007; 25: 1055-1063.

37. Kesler K, et al. Immune responses of prematurely born infants to hepatitis B vaccination: results through three years of age. Paediatric Infectious Disease Journal 1998; 17: 116-119.

38. Losonsky GA, et al. Hepatitis B vaccination of premature infants: A reassessment of current recommendations for delayed immunization. Pediatrics 1999; 103: e14.
39. Lau YL, et al. Response of preterm infants to hepatitis B vaccine. Journal of Pediatrics 1992; 121 : 962-965.

40. Olivier C, et al. Immunogenicity, reactogenicity, and safety of a seven-valent pneumococcal conjugate vaccine (PCV7) concurrently administered with a fully liquid DTPa-IPV-HBV-Hib combination vaccine in healthy infants. Vaccine 2008; 26: 3142-3152.

41. Tichmann-Schumann I, et al. Immunogenicity and reactogenicity of four doses of diphtheria-tetanus threecomponent acellular pertussis-hepatitis B-inactivated polio virus Haemophilus influenzae type $b$ vaccine coadministered with 7-valent pneumococcal conjugate vaccine. Pediatric Infectious Disease Journal 2005; 24: $70-77$.

42. Da Villa G, et al. Impact of hepatitis B vaccination in a highly endemic area of south Italy and long-term duration of anti-HBs antibody in two cohorts of vaccinated individuals. Vaccine 2007; 25: 3133-3136.

43. Bialek SR, et al. Persistence of protection against hepatitis $\mathrm{B}$ virus infection among adolescents vaccinated with recombinant hepatitis $\mathrm{B}$ vaccine beginning at birth: a 15-year follow-up study. Pediatric Infectious Disease Journal 2008; 27 : 881-885.

44. Whittle H, et al. Observational study of vaccine efficacy 14 years after trial of hepatitis B vaccination in Gambian children. British Medical Journal 2002; 14: 569-573.

45. Samandari T, et al. Differences in response to a hepatitis $B$ vaccine booster dose among Alaskan children and adolescents vaccinated during infancy. Pediatrics 2007; 120: e373-e381.

46. Walter J, et al. Hepatitis B and C risk factors in Germany-results of the national surveillance [in German]. Gesundheitswesen 2005; 67: 441-447.

47. Swissmedic. Notification to health experts, 20 September 2005: Swissmedic suspends the license of the Hexavac vaccine [in German]. Swissmedic Journal 2005; 9: 678-679.

48. WHO. International Classification of Diseases (ICD), 2007. World Health Organization (http://apps.who. int/classifications/apps/icd/icd10online/). Accessed 30 October 2009. 